\title{
Heat and Mass Transfer on MHD Jeffrey-Hamel Flow in Presence of Inclined Magnetic Field
}

\author{
Edward Richard Onyango ${ }^{1, ~}$, Mathew Ngugi Kinyanjui ${ }^{1}$, Mark Kimathi ${ }^{1,2}$, \\ Surindar Mohan Uppal ${ }^{1}$ \\ ${ }^{1}$ Department of Pure and Applied Mathematics, Jomo Kenyatta University of Agriculture and Technology, Nairobi, Kenya \\ ${ }^{2}$ Department of Mathematics, Statistics and Actuarial Science, Machakos University, Machakos, Kenya
}

Email address:

edwardrichard36@gmail.com (E. R. Onyango)

${ }^{*}$ Corresponding author

\section{To cite this article:}

Edward Richard Onyango, Mathew Ngugi Kinyanjui, Mark Kimathi, Surindar Mohan Uppal. Heat and Mass Transfer on MHD JeffreyHamel Flow in Presence of Inclined Magnetic Field. Applied and Computational Mathematics. Vol. 9, No. 4, 2020, pp. $108-117$. doi: 10.11648/j.acm.20200904.11

Received: May 11, 2020; Accepted: June 3, 2020; Published: June 17, 2020

\begin{abstract}
In this study, a magnetohydrodynamic Jeffrey-Hamel flow of a viscous, fluid that conducts electricity and is incompressible through a divergent conduit in presence of inclined variable magnetic field with heat and mass transfer has been investigated. The solutions of the governing equations of the MHD flow are obtained numerically since they are nonlinear. The numerical scheme used is implemented in a computer software program and the results presented in graphical form. The velocity profile, the temperature profiles, the effect of variable magnetic field and of varying various dimensionless numbers on the flow are analyzed. Jeffrey-Hamel flows are also applied in the diffuser development. Some of the systems include; the channel between the compressor and gas turbine engine burner, the canal at departure from a gas turbine linked to the jet pipe, the canal subsequent to the impellor of a centrifugal compressor, wind tunnels with closed circuits, and water turbine draft tubes among several others. The results provide significant information for the improvement of proficiency and performance of technologies in aerospace, chemical, civil, environmental, industrial and mechanical applications.
\end{abstract}

Keywords: Unsteadiness Parameter, Viscous Dissipation, Inclined Variable Magnetic Field

\section{Introduction}

Magnetohydrodynamics (MHD) describes the intricate interaction between magnetic fields and plasmas that are accountable for considerable dynamic conduct in various cosmic matters including the sun. MHD is significant in planetary processes such as magneto-convection, magnetic flux occurrence, flux ropes, spots, atmospheric heating, wind acceleration, flares, and eruptions. Mass transfer is the transport of one constituent from a region of higher concentration to that of a lower concentration from a system that contains two or more components whose concentrations vary from point to point. Heat transfer rate depends on the systems temperature and the properties of the medium intervening through which the transfer of heat takes place. Two-dimensional steady motion of a viscous fluid through divergent-convergent channels which is referred to commonly to as the classical Jeffery-Hamel flow in fluid dynamics was first studied by Jeffery [1] and Hamel [2]. The flow models for Jeffery-Hamel flows are interesting and are used to demonstrate the phenomenon of boundary layers separation in divergent channels. Jeffrey and Hamel developed a solutions for the Navier-Stokes equation using the similarity concept that depended on two parameters that were non-dimensional which were the flow Reynolds number and the angle of the channels widths. The classical JefferyHamel problem was further analyzed by Axfold [3] studying the effects of the magnetic field that is external to the conducting fluid. He concluded that the magnetic field acted as a control parameter as well as the Reynolds number for the flow and the angle of the walls. The study [4] reduced the Maxwell's electromagnetism governing equations and the Navier-Stokes equations to ordinary differential equations that were nonlinear for the model the problem of the JefferyHamel flow for a case where the magnetic field was high in 
the presence of nanoparticles. The flow region in the channel that was divergent was studied with different values of Hartmann number and different values of the angle of channel and their results when matched with the exact solution obtained by Adomian's Decomposition Method (ADM) were in agreement.[5] while studying the flow over convergent and divergent wall rib-lets conducted an experiment in the research laboratory of the High-Speed wind Tunnel of the Dresden University of Technology (Germany) to determine the velocity field over convergent and divergent rib-let patterns by hot-wire measurements in turbulent pipe flow. They concluded that adjacent to the wall of the channel, convergent and divergent rib-let patterns show considerable differences with regard to the timeaveraged stream-wise velocity and the stream-wise velocity fluctuations. If the rib-lets converge, the average time velocity decreased while velocity fluctuations increased with the opposite for divergent rib-let patterns. Magnetohydrodynamic flows which was an extension of the classical Jeffrey-Hamel flows in divergent and convergent channels Jeffery-Hamel flows to MHD interpreting the effect of the external magnetic field working as a parameter in the solution of the channel flows for the divergent and convergent channels was analyzed by the study [6]. MHD flows in convergent- divergent channels studies extended from the classical Jeffery-Hamel flows to the MHD studies done by the study [6] was studied by the study [7] introducing the method of Adomian decomposition and determining the Adomian's polynomial. They obtained a solution for the problem for the case of divergent and the case of convergent channels concluding that the velocity distribution on the fluid flow and the shear stress constant is depicted at various Reynolds numbers. They compared their results with some earlier works which illustrated their excellent accuracy.[8] discussed slip and Joule heating effects in mixed convection peristaltic transport of nanofluid with Soret and Dufour effects. The study [9] considered blowing/suction effect on hydromagnetic heat transfer by mixed convection from an inclined continuously stretching surface with internal heat generation/absorption. The study [10] Examined double-diffusive convection in a porous enclosure with cooperating temperature and concentration gradients and heat generation or absorption effects.Unsteady MHD free convective visco-elastic fluid flow bounded by an infinite inclined porous plate with a heat source, viscous dissipation, and Ohmic heating was investigated by the study [11]. Motivated by the above investigations, in this work a fully developed free convective flow of a viscous incompressible electrically conducting fluid past a vertical porous plate bounded by a porous medium in the presence of thermal radiation, heat source/sink, variable suction, and variable permeability is analyzed. The study [12] studied the MHD boundary layer flow of a VISCO-Elastic fluid past a porous plate with varying suction and heat source/sink in the presence of thermal radiation and diffusion. They considered a case of a magnetic field whose strength was uniform and perpendicular to the plate with heat source. They concluded in this study that the presence of thermal radiation decreases the temperature, an opposite nature is shown in the case of Eckert number and the influence of the heat source leads to enhance the temperature.

Jeffery-Hamel flows with heat transfer of nanofluids using the homotopy perturbation method and comparing with numerical results were studies by the study [13]. They considered the influence of nanoparticles on the nonlinear Jeffery-Hamel flow problem investigating three types of nanoparticles namely Copper, Alumina and Titania by considering water as a base fluid. They concluded that the effect of solid volume fraction of nanoparticles on the heat transfer and fluid flow parameters is more pronounced when compared with the type of nanoparticles and the skin friction coefficient and Nusselt number for alumina nanofluid is the highest in comparison to the other two nanoparticles.

Analysis of heat and mass transfer for unsteady viscous MHD nanofluid flowing through a conduit whose walls are permeable in presence of metal nanoparticles was done by [14]. They considered two cases for effective thermal conductivity through the $\mathrm{H}-\mathrm{C}$ model and concluded that the permeability of the conduit increased shear stress at lower wall. Heat transfer rate increases with the increase of the Reynolds number and Mass transfer rate decreases with the increase of Reynolds number thermal boundary layer thickness is a decreasing function when injection/suction happens altogether on HTP.

A Jeffery-Hamel flow of non-Newtonian Micropolar incompressible fluid inside non-parallel walls and notices heat transfer effect in flow region was studied by the study [15]. They converted the governing nonlinear PDEs to nonlinear coupled ODEs using appropriate similarity transformations and solved them with the utilization of the Taylor optimization method based on differential evolution (DE) algorithm. They concluded from their results that the fluid velocity was decreased, although the angular velocity of micro constituents and heat transfer in the flow was increased as enlarging the values of the vortex viscosity parameter associated with the divergent channel. It also noticed that both spin-gradient viscosity and micro-inertia density enhance the micro rotation profiles and their results agreed with the results obtained by the fourth-order Runge-Kutta method.

Magnetohydrodynamics Jeffery-Hamel flow with heat transfer problem in an Eyring-Powell fluid using differential transform method was studied by the study [16] where they analyzed the variations of velocity profiles for different values of the Reynolds number, Eckert number, Prandtl number and Hartmann number in the flow with heat transfer with the fluid in both divergent and convergent channels and concluded that the nanofluid flow velocity profile increases as the value of the Eckert number and the Prandtl number increases and decreases as the value of the Hartmann number increases, on the contrary, the heat profile fluid flow also increases. They also deduced that the velocity profile of Jeffrey Hamel nano-fluid flow decreases as the value of Reynolds number increases. 
More recently, The study [17] studied the unsteady twodimensional Jeffery-Hamel flow of an incompressible nonNewtonian fluid, with nonlinear viscosity and skin friction, flowing through a divergent channel in the presence of a magnetic field in the direction perpendicular to the motion of the fluid. They noted that when the Reynolds number and Hartmann number was increased, the velocity of the fluid increased. However, when the unsteadiness parameter was increased, the velocity of the fluid decreased and was a constant when the values of the Eckert number and the Prandtl number were increased. The temperature of the fluid increased when the Reynolds, Hartmann, Prandtl and Eckert number were increased with the same case for the unsteadiness parameter.

\section{Mathematical Formulation}

The analysis of unsteady Jeffrey Hamel MHD flows between porous walls with injection or suction where there is a magnetic field that is oblique with heat and mass transfer has not been extensively analyzed. This research is focused on a 2D unsteady Jeffrey Hamel MHD incompressible viscous fluid that conducts electricity flowing from a source at the connection between two porous walls with a case where there is injection / suction with an inclined magnetic field that is variable, with the walls at an angle $2 \alpha$ as in figure 1. If $\alpha>0$, the walls are convergent and divergent if $\alpha<0$. The walls are considered to be rigid. The velocity of the fluid is considered to be along the radial direction. The magnetic field is inclined at an angle of $\beta$ as shown in the geometrical model illustration of the problem in figure 1 .

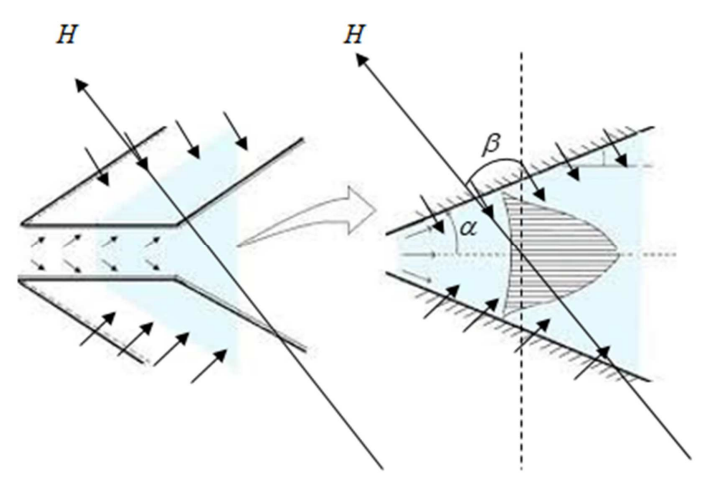

Figure 1. Geometrical illustration.

The total angle between the walls is $\theta$ such that $-\alpha \leq \theta \leq \alpha$ and $\theta=2 \alpha$. The suction velocity or the injection velocity is a constant $u_{0} . T_{\infty}$ and $T_{w}$ where $T_{\infty}>T_{w}$ are the free stream and wall temperatures respectively while $C_{\infty}$ and $C_{w}$ are the free stream and concentrations at walls with $C_{\infty}<C_{w}$.

\section{Governing Equations}

In cylindrical coordinate system, the general equations for the flow is given by

$$
\begin{gathered}
\frac{\partial \rho}{\partial t}+\frac{1}{r} \frac{\partial}{\partial r}\left(r \rho u_{r}\right)+\frac{1}{r} \frac{\partial}{\partial \theta}\left(\rho u_{\theta}\right)+\frac{\partial}{\partial z}\left(\rho u_{z}\right)=0 \\
\rho\left(\frac{\partial u_{r}}{\partial t}+u_{r} \frac{\partial u_{r}}{\partial r}+\frac{u_{\theta}}{r} \frac{\partial u_{r}}{\partial \theta}-\frac{u_{\theta}^{2}}{r}+u_{z} \frac{\partial u_{r}}{\partial z}\right)=-\frac{\partial p}{\partial r}+\mu\left(\frac{1}{r} \frac{\partial}{\partial r}\left(\frac{\partial\left(r u_{r}\right)}{\partial r}\right)+\frac{1}{r^{2}}\left(\frac{\partial^{2} u_{r}}{\partial \theta^{2}}\right)-\frac{2}{r^{2}} \frac{\partial u_{\theta}}{\partial \theta}+\frac{\partial^{2} u_{r}}{\partial z^{2}}\right)+\rho F_{r} \\
\rho\left(\frac{\partial u_{\theta}}{\partial t}+u_{r} \frac{\partial u_{\theta}}{\partial r}+\frac{u_{\theta}}{r} \frac{\partial u_{\theta}}{\partial \theta}+\frac{u_{r} u_{\theta}}{r}+u_{z} \frac{\partial u_{\theta}}{\partial z}\right)=-\frac{1}{r} \frac{\partial p}{\partial \theta}+\mu\left(\frac{1}{r} \frac{\partial}{\partial r}\left(\frac{\partial\left(r u_{\theta}\right)}{\partial r}\right)+\frac{1}{r^{2}}\left(\frac{\partial^{2} u_{\theta}}{\partial \theta^{2}}\right)+\frac{2}{r^{2}} \frac{\partial u_{r}}{\partial \theta}+\frac{\partial^{2} u_{\theta}}{\partial z^{2}}\right)+\rho F_{\theta} \\
\left.\frac{\partial C}{\partial t}+\frac{d T}{d t}+u_{r} \frac{\partial T}{\partial r}+\frac{u_{\theta}}{r} \frac{\partial T}{\partial \theta}+u_{z} \frac{\partial T}{\partial z}\right)=\mathrm{K}\left[\frac{1}{r} \frac{\partial}{\partial r}\left(r \frac{\partial T}{\partial r}\right)+\frac{1}{r^{2}} \frac{\partial^{2} \mathrm{~T}}{\partial \theta^{2}}+\frac{\partial^{2} \mathrm{~T}}{\partial z^{2}}\right]+\Phi \\
\partial C
\end{gathered}
$$

$$
\frac{\partial \mathbf{H}}{\partial t}=\frac{1}{\mu_{e} \sigma}\left(\nabla^{2} \mathbf{H}\right)+\frac{1}{\mu_{e}}(\nabla \times[\mathbf{V} \times \mathbf{B}])
$$

Using the Maxwell equations to obtain the Lorentz force by using $\mathbf{J} \times \mathbf{B}$. The total electric field $\mathbf{J}$ from the Ohms law is given by

$$
\mathbf{J}=\sigma(\mathbf{V} \times \mathbf{B})
$$

The velocity of the fluid and the magnetic field resulting from the induction are given by $\mathbf{V}=u_{r} \cos \theta \hat{r}+0 \hat{\theta}+0 \hat{z}$, and $\mathbf{B}=\left(H_{r}-H \sin \beta\right) \hat{r}+\left(H_{\theta}+H \cos \beta\right) \hat{\theta}+0 \hat{z}$ respectively.

With the flow purely along the $r$ and $\theta$ directions and considering no changes in the $z$ direction and applying Boussinesq approximation, taking into account Joule heating and viscous dissipation for a case of no chemical reaction, the specific equations of the flow are obtained as; 


$$
\begin{aligned}
& \frac{1}{r} \frac{\partial}{\partial r}\left(r u_{r}(r, \theta)\right)=0 \\
& \hat{r}: \rho\left(\frac{\partial u_{r}}{\partial t}+u_{r} \frac{\partial u_{r}}{\partial r}-\frac{u_{\theta}^{2}}{r}+\frac{u_{\theta}}{r} \frac{\partial u_{r}}{\partial \theta}\right)=-\frac{\partial p}{\partial r}+\mu\left(\frac{1}{r} \frac{\partial}{\partial r}\left(\frac{\partial\left(r u_{r}\right)}{\partial r}\right)+\frac{1}{r^{2}}\left(\frac{\partial^{2} u_{r}}{\partial \theta^{2}}\right)\right)-\left(u_{r} \sigma\left(H_{\theta}+H \cos \beta\right)^{2} \cos \theta\right) \\
& \hat{\theta}: \rho\left(\frac{\partial u_{\theta}}{\partial t}+u_{r} \frac{\partial u_{\theta}}{\partial r}+\frac{u_{r} u_{\theta}}{r}\right)=-\frac{1}{r} \frac{\partial p}{\partial \theta}+\mu\left(\frac{1}{r} \frac{\partial}{\partial r}\left(\frac{\partial\left(r u_{\theta}\right)}{\partial r}\right)+\frac{2}{r^{2}} \frac{\partial u_{r}}{\partial \theta}\right)+\left(u_{r} \sigma\left(H_{r}-H \sin \beta\right)\left(H_{\theta}+H \cos \beta\right) \cos \theta\right)+\rho g\left[\beta_{*}\left(T-T_{\infty}\right)+\beta_{c}\left(C-C_{\infty}\right)\right] \\
& \rho C_{p}\left(\frac{d T}{d t}+u_{r} \frac{\partial T}{\partial r}+\frac{u_{\theta}}{r} \frac{\partial T}{\partial \theta}\right)=\mathrm{K}\left(\frac{1}{r} \frac{\partial T}{\partial r}+\frac{\partial^{2} T}{\partial r^{2}}+\frac{1}{r^{2}} \frac{\partial^{2} \mathrm{~T}}{\partial \theta^{2}}\right)+\mu\left[2\left(\frac{\partial u_{r}}{\partial r}\right)^{2}+2\left(\frac{u_{r}}{r}\right)^{2}+\left(-\frac{u_{\theta}}{r}\right)^{2}-\frac{2 u_{\theta}}{r^{2}} \cdot\left(\frac{\partial u_{r}}{\partial \theta}\right)+\frac{1}{r^{2}}\left(\frac{\partial u_{r}}{\partial \theta}\right)^{2}\right] \\
& +\sigma\left(u_{r}\left(H_{\theta}+H \cos \beta\right) \cos \theta\right)^{2} \\
& \frac{\partial C}{\partial t}+u_{r} \frac{\partial C}{\partial r}+u_{\theta} \frac{\partial C}{\partial \theta}=D\left(\frac{1}{r} \frac{\partial C}{\partial r}+\frac{\partial^{2} C}{\partial r^{2}}+\frac{1}{r^{2}} \frac{\partial^{2} C}{\partial \theta^{2}}\right) \\
& \hat{r}: \frac{\partial H_{r}}{\partial t}=\frac{1}{\mu_{e} \sigma}\left(\frac{\partial^{2} H_{r}}{\partial r^{2}}+\frac{1}{r} \frac{\partial H_{r}}{\partial r}+\frac{1}{r^{2}} \frac{\partial^{2} H_{r}}{\partial \theta^{2}}\right)-\frac{u_{r} \sigma}{r} \frac{1}{\mu_{e}}\left(H_{\theta} \sin \theta+H \cos \beta \sin \theta\right) \\
& \hat{\theta}: \frac{\partial H_{\theta}}{\partial t}=\frac{1}{\mu_{e} \sigma}\left(\frac{\partial^{2} H_{\theta}}{\partial r^{2}}+\frac{1}{r} \frac{\partial H_{\theta}}{\partial r}+\frac{1}{r^{2}} \frac{\partial^{2} H_{\theta}}{\partial \theta^{2}}\right)+\frac{\sigma}{\mu_{e}}\left[\frac{\partial u_{r}}{\partial r}\left(H_{\theta}+H \cos \beta\right) \cos \theta+\frac{u_{r}}{r}\left(H_{\theta}+H \cos \beta\right) \cos \theta\right]
\end{aligned}
$$

Obtaining the difference of the differentiated equation (9) w.r.t $\hat{\theta}$ and multiplied equation (10) by $\hat{r}$ and differentiated w.r.t $\hat{r}$ the momentum equation is obtained in cylindrical form.

$$
\begin{aligned}
& \frac{\partial^{2} u_{r}}{\partial t \partial \theta}+\frac{\partial u_{r}}{\partial \theta} \cdot \frac{\partial u_{r}}{\partial r}+u_{r} \frac{\partial^{2} u_{r}}{\partial \theta \partial r}+\frac{u_{\theta}}{r} \frac{\partial^{2} u_{r}}{\partial \theta^{2}}-u_{\theta} \frac{\partial u_{r}}{\partial r} \\
& =\frac{\mu}{\rho}\left(\begin{array}{l}
\left.\frac{1}{r} \frac{\partial}{\partial \theta}\left(\frac{\partial u_{r}}{\partial r}\right)-\frac{u_{r}}{r^{2}}+\frac{\partial}{\partial \theta}\left(\frac{\partial^{2} u_{r}}{\partial r^{2}}\right)+\frac{1}{r^{2}}\left(\frac{\partial^{3} u_{r}}{\partial \theta^{3}}\right)-\frac{u_{\theta}}{r^{2}}+\frac{2}{r^{2}} \frac{\partial u_{r}}{\partial \theta}\right)+u_{r}\left(\frac{1}{\rho} \sigma\left(H_{\theta}+H \cos \beta\right)^{2} \sin \theta\right)-\frac{\partial u_{r}}{\partial \theta}\left(\frac{1}{\rho} \sigma\left(H_{\theta}+H \cos \beta\right)^{2} \cos \theta\right) \\
-\frac{2}{r} \frac{\partial}{\partial \theta}\left(\frac{\partial u_{r}}{\partial r}\right)
\end{array}\right) \\
& -\left(u_{r}+r \frac{\partial u_{r}}{\partial r}\right) \frac{\sigma}{\rho}\left(\left(H_{r}-H \sin \beta\right)\left(H_{\theta}+H \cos \beta\right) \cos \theta\right)-g\left[\beta_{*}\left(T-T_{\infty}\right)+\beta_{c}\left(C-C_{\infty}\right)\right]
\end{aligned}
$$

Equations (8), (11), (12), (13), (14) and (15) are specific equations governing the flow.

Introducing the unsteadiness parameter and the wedge angle parameter $\quad \lambda=\frac{\delta^{m}}{v r^{m-1}} \frac{d \delta}{d t} \quad$ and $\quad \alpha=\frac{m}{m+1} \pi$ respectively to account for unsteadiness and the wedge angle size from studies done by the studies $[15,16,19,20,22]$, and [23] where $\delta$ is a function of $t$ is a time-dependent length scale whereas the parameter $m$ is linked to angle of the wedge together with wedge radius.

\section{Numerical Solution}

Similarity transformation is applied in reducing the governing equations to ordinary differential equations that are further reduced to degree one before applying the collocation method.
From research work done by the studies $[15,16,19]$, and other scholars, the following transformations are used.

$$
\begin{gathered}
u_{r}(\theta, t)=-\frac{Q}{r} \frac{1}{\delta^{m+1}} \eta(\theta)=-\frac{Q}{r} \frac{1}{\delta^{m+1}} \eta \\
\frac{\psi(\theta)}{\delta^{m+1}}=\frac{T-T_{\infty}}{T_{w}-T_{\infty}}, \\
\frac{\phi(\theta)}{\delta^{m+1}}=\frac{C-C_{\infty}}{C_{w}-C_{\infty}}, \\
H_{\theta}(\theta, t)=-\frac{Q}{r} \frac{1}{\delta^{m+1}} \gamma(\theta)=-\frac{Q}{r} \frac{1}{\delta^{m+1}} \gamma
\end{gathered}
$$

Applying the transformations on the governing equations, the following ordinary differential equations are obtained; 


$$
\begin{aligned}
& r^{2} \frac{m+1}{\delta v} \frac{d \delta}{d t} \eta^{\prime}-2 Q \frac{1}{v \delta^{m+1}} \eta \eta^{\prime}-\frac{r u_{\theta}}{v}\left(\eta^{\prime \prime}+\eta\right)=\eta-5 \eta^{\prime}-\eta^{\prime \prime \prime}-\frac{r u_{\theta} \delta^{m+1}}{Q}-r^{2} \eta \frac{\sigma}{\mu}\left(H_{\theta}+H \cos \beta\right)^{2} \sin \theta \\
& +r^{2} \eta^{\prime} \frac{\sigma}{\mu}\left(H_{\theta}+H \cos \beta\right)^{2} \cos \theta-g \frac{\beta_{*} r^{3}\left(T_{w}-T_{\infty}\right) \omega}{v Q}-g \frac{\beta_{c} r^{3}\left(C-C_{\infty}\right) \phi}{v Q} \\
& \rho C_{p}\left(-\frac{m+1}{\delta^{m+2}} \frac{d \delta}{d t}\left(T_{w}-T_{\infty}\right) \psi+\frac{u_{\theta}}{r} \cdot \frac{T_{w}-T_{\infty}}{\delta^{m+1}} \psi^{\prime}\right)=\mathrm{K}\left(\frac{1}{r^{2}} \cdot \frac{T_{w}-T_{\infty}}{\delta^{m+1}} \psi^{\prime \prime}\right) \mu\left[\begin{array}{l}
2\left(\frac{Q}{r^{2}} \frac{1}{\delta^{m+1}} \eta\right)^{2}+2\left(-\frac{Q}{r^{2}} \frac{1}{\delta^{m+1}} \eta\right)^{2}+\left(-\frac{u_{\theta}}{r}\right)^{2}- \\
\frac{2 u_{\theta}}{r^{2}} \cdot\left(-\frac{Q}{r} \frac{1}{\delta^{m+1}} \eta^{\prime}\right)+\frac{1}{r^{2}}\left(-\frac{Q}{r} \frac{1}{\delta^{m+1}} \eta^{\prime}\right)^{2}
\end{array}\right] \\
& +\sigma\left(\left(-\frac{Q}{r} \frac{1}{\delta^{m+1}} \eta\right)\left(H_{\theta}+H \cos \beta\right) \cos \theta\right)^{2} \\
& -\frac{m+1}{\delta^{m+2}} \frac{d \delta}{d t}\left(C_{w}-C_{\infty}\right) \phi+\left(-\frac{Q}{r} \frac{1}{\delta^{m+1}} \eta\right)(0)+u_{\theta} \frac{C_{w}-C_{\infty}}{\delta^{m+1}} \phi^{\prime}=D\left(\frac{1}{r}(0)+(0)+\frac{1}{r^{2}} \frac{C_{w}-C_{\infty}}{\delta^{m+1}} \phi^{\prime \prime}\right) \\
& -\frac{m+1}{\delta^{m+2}} \frac{Q}{r} \frac{d \delta}{d t} \gamma=\frac{1}{\mu_{e} \sigma}\left(\begin{array}{l}
-\frac{2 Q}{r^{3}} \frac{1}{\delta^{m+1}} \gamma+\frac{Q}{r^{3}} \frac{1}{\delta^{m+1}} \gamma \\
-\frac{Q}{r^{3}} \frac{1}{\delta^{m+1}} \gamma^{\prime \prime}
\end{array}\right)+\frac{Q}{r^{2}} \frac{1}{\delta^{m+1}} \eta \frac{\sigma}{\mu_{e}}\left(H_{\theta}+H \cos \beta\right) \sin \theta
\end{aligned}
$$

Applying the following dimensionless numbers

$$
\begin{aligned}
& R_{e}=\frac{r u_{\theta}}{v}=\frac{Q}{v r}, H_{a}=r^{2} \frac{\sigma}{\mu}\left(H_{\theta}+H \cos \beta\right)^{2}, G_{r(T)}=\frac{g \beta_{*} r^{3}\left(T_{w}-T_{\infty}\right)}{v Q}, G_{r(C)}=\frac{g \beta_{c} r^{3}\left(C-C_{\infty}\right)}{v Q}, P_{r}=\frac{\mu C_{p}}{\mathrm{~K}}, R_{e}=\frac{r \cdot u_{\theta}}{v}, R_{e}=\frac{r \cdot u_{\theta}}{v}, \\
& S_{c}=\frac{v}{D}, E_{c}=\frac{Q^{2}}{r^{2} C_{p}\left(T_{w}-T_{\infty}\right)}, J=\frac{\sigma\left(H_{\theta}+H \cos \beta\right)^{2}}{\rho C_{p} v\left(T_{w}-T_{\infty}\right)}, R_{e}=\frac{r u_{\theta}}{v} \cdot R_{m}=\sigma \mu_{e} r u_{\theta}, \text { and } H_{a}=r\left(\frac{\sigma}{\mu_{e}}\right)^{1 / 2}\left(H_{\theta}+H \cos \beta\right)
\end{aligned}
$$

to equation (20), (21), (22) and (23) and rearranging the equations.

$$
\begin{gathered}
\eta^{\prime \prime \prime}=R_{e}\left(\eta^{\prime \prime}+\eta\right)+2 r R_{e} \frac{1}{\delta^{m+1}} \eta \eta^{\prime}+f-5 \eta^{\prime}-\frac{r u_{\theta} \delta^{m+1}}{Q}-H_{a}^{2} \eta \sin \theta+H_{a}^{2} \eta^{\prime} \cos \theta-G_{r(T)} \omega-G_{r(C)} \phi-(m+1) \frac{\delta^{m+1}}{r^{m+1}} \lambda \eta^{\prime} \\
\psi^{\prime \prime}=-\frac{m+1}{\delta^{m+1}} r^{m+1} P_{r} \lambda \psi+P_{r} R_{e} \psi^{\prime}-P_{r} \cdot E_{c}\left[4 \frac{1}{\delta^{m+1}} \eta^{2}+\frac{r^{2} u_{\theta}^{2}}{Q^{2}} \delta^{m+1}+\frac{2 r u_{\theta}}{Q} \eta^{\prime}+\frac{1}{\delta^{m+1}} \eta^{\prime 2}\right]-Q^{2} P_{r} J \frac{1}{\delta^{m+1}} \eta^{2}\left(\cos ^{2} \theta\right) \\
\phi^{\prime \prime}=r \mathrm{R}_{\mathrm{e}} \cdot S_{c} \phi^{\prime}-\frac{m+1}{\delta^{m+1}} r^{m+1} \lambda \cdot S_{c} \phi \\
\gamma^{\prime \prime}=r^{m+2} \frac{m+1}{\delta^{m+1}} \lambda \frac{R_{m}}{R_{e}} \gamma-\gamma+\frac{1}{v} \sqrt{\left(\frac{\sigma}{\mu_{e}}\right)} H_{a} \frac{R_{m}}{R_{e}} \eta \sin \theta
\end{gathered}
$$

Equations (24-27), are the ODEs prevailing the fluid flow.

Transforming the conditions on the boundary by the same similarity transforms;

For $\theta=0, \eta(0)=-\frac{u_{\infty} r}{Q} \delta^{m+1} u_{\theta}=0, \psi(0)=0, \phi(0)=0$

$$
\text { For } \theta= \pm \alpha, \eta( \pm \alpha)=0, u_{\theta}=u_{0}, \psi( \pm \alpha)=\delta^{m+1}, \phi(0)=\delta^{m+1}
$$

Equations (24-27) are reduced to ODEs of order one and solved. Letting

$$
x_{1}=\eta, x_{2}=\eta^{\prime}, x_{3}=\eta^{\prime \prime}, x_{4}=\psi, x_{5}=\psi^{\prime}, x_{6}=\phi, x_{7}=\phi^{\prime}, x_{8}=\gamma, x_{9}=\gamma^{\prime}
$$


Getting the derivatives of (29)

$$
\begin{aligned}
& x_{1}^{\prime}=x_{2} \\
& x_{2}^{\prime}=x_{3} \\
& x_{3}^{\prime}=\eta^{\prime \prime \prime}=R_{e}\left(x_{3}+x_{1}\right)+2 r R_{e} \frac{1}{\delta^{m+1}} x_{1} x_{2}+x_{1}-5 x_{2}-\frac{r u_{\theta} \delta^{m+1}}{Q}-H_{a}{ }^{2} x_{1} \sin \theta+H_{a}{ }^{2} x_{2} \cos \theta-G_{r(T)} \omega-G_{r(C)} \phi-(m+1) \frac{\delta^{m+1}}{r^{m+1}} \lambda x_{2} \\
& x_{4}^{\prime}=x_{5} \\
& x_{5}^{\prime}=\psi^{\prime \prime}=-\frac{m+1}{\delta^{m+1}} r^{m+1} P_{r} \lambda x_{4}+P_{r} R_{e} x_{5}-P_{r} \cdot E_{c}\left[4 \frac{1}{\delta^{m+1}} x_{1}^{2}+\frac{r^{2} u_{\theta}^{2}}{Q^{2}} \delta^{m+1}+\frac{2 r u_{\theta}}{Q} x_{2}+\frac{1}{\delta^{m+1}} x_{2}^{2}\right]-Q^{2} P_{r} J \frac{1}{\delta^{m+1}} x_{1}^{2}\left(\cos ^{2} \theta\right) \\
& x_{6}^{\prime}=x_{7} \\
& x_{7}^{\prime}=\phi^{\prime \prime}=r \mathrm{R}_{\mathrm{e}} \cdot S_{c} x_{7}-\frac{m+1}{\delta^{m+1}} r^{m+1} \lambda . S_{c} x_{6} \quad \text { (iii) } \\
& x_{8}^{\prime}=x_{9} \\
& x_{9}^{\prime}=\gamma^{\prime \prime}=r^{m+2} \frac{m+1}{\delta^{m+1}} \lambda \frac{R_{m}}{R_{e}} x_{8}-x_{8}+\frac{1}{v} \sqrt{\left(\frac{\sigma}{\mu_{e}}\right)} H_{a} \frac{R_{m}}{R_{e}} x_{1} \sin \theta \quad \text { (iv) }
\end{aligned}
$$

A general form of vectors is used to represent equations (29-35)

$$
\begin{gathered}
x^{\prime}=F(\theta, x) \\
\text { With vectors }\left(\begin{array}{c}
x_{1} \\
x_{2} \\
x_{3} \\
x_{4} \\
x_{5} \\
x_{6} \\
x_{7} \\
x_{8} \\
x_{9}
\end{array}\right) \text { and } F=\left(\begin{array}{c}
x_{2} \\
x_{3} \\
(i) \\
y_{5} \\
(i i) \\
x_{7} \\
(i i i) \\
x_{9} \\
(i v)
\end{array}\right)
\end{gathered}
$$

The collocation method together with the implicit RungeKutta method inbuilt in MATLAB as a function known as the bvp4c is used to solve equations (39).

\section{Results and Discussion}

The following graphs illustrate the effects of several flow parameters and variables.

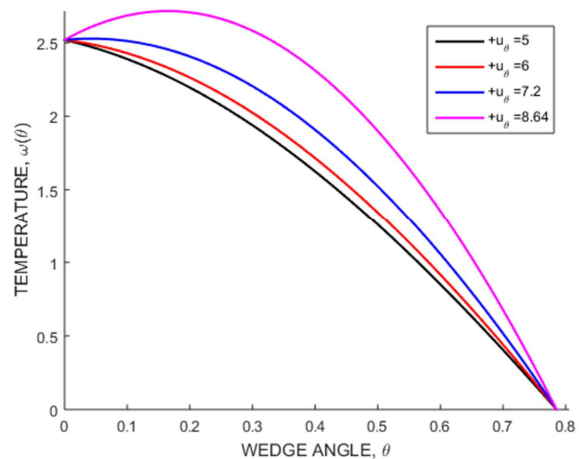

Figure 2. Temperature profiles for different values of the suction parameter.

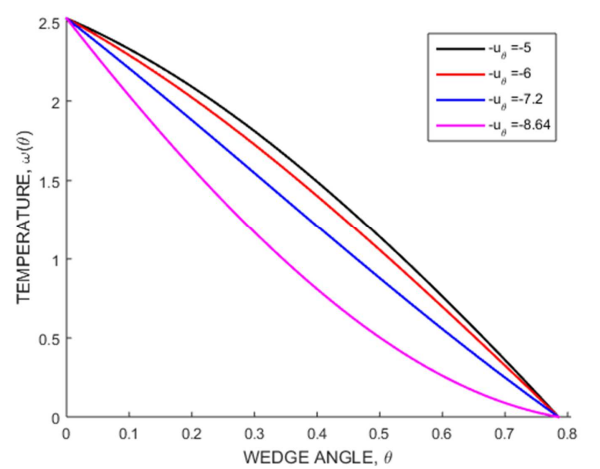

Figure 3. Temperature profiles for different values of the injection parameter. 


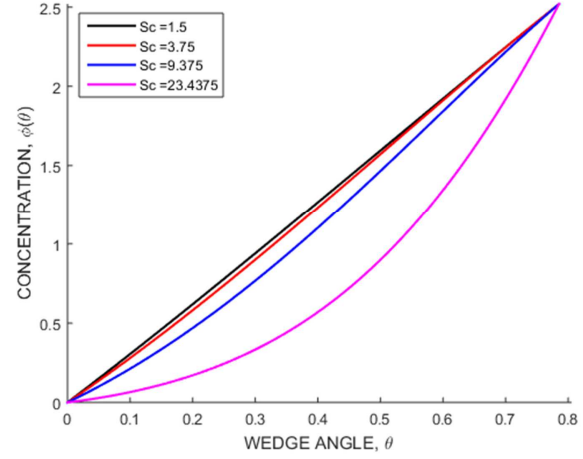

Figure 4. Concentration profiles for different values of the Schmidt number.

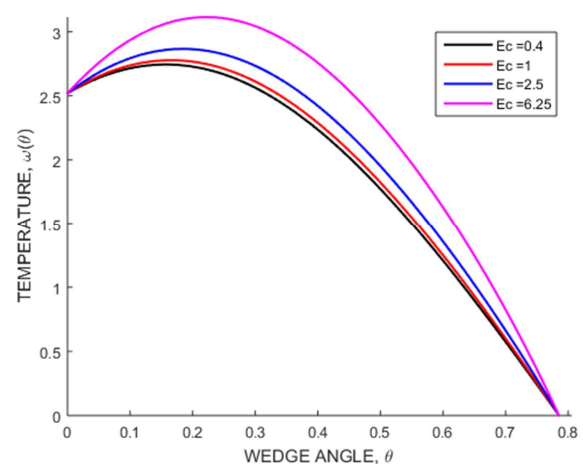

Figure 5. Temperature profiles for different values of the Eckert number.

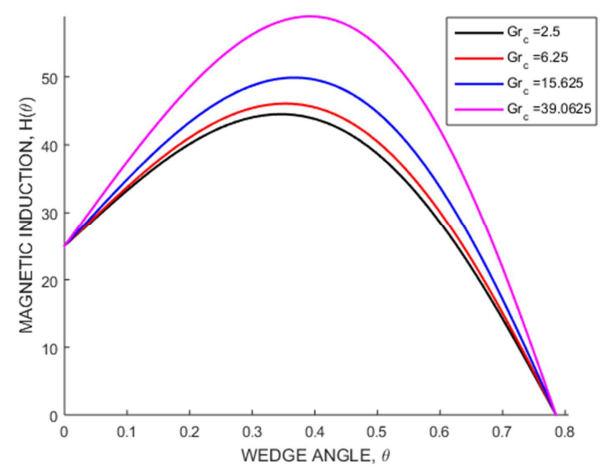

Figure 6. Magnetic Induction profiles for different values of the Grashof Concentration number.

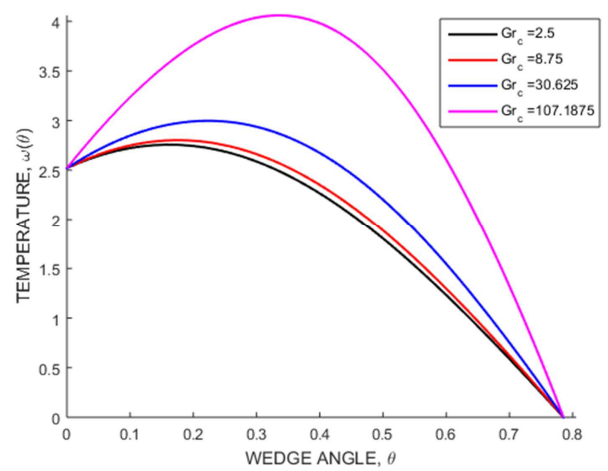

Figure 7. Temperature profiles for different values of the Grashof Concentration number.

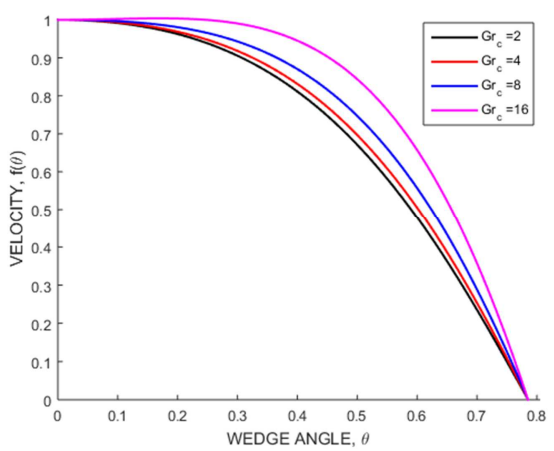

Figure 8. Velocity profiles for different values of the Grashof Concentration number.

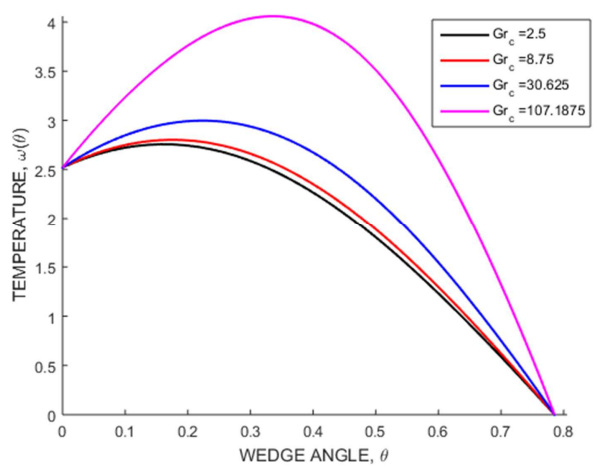

Figure 9. Velocity profiles for different values of the Grashof Temperature number.

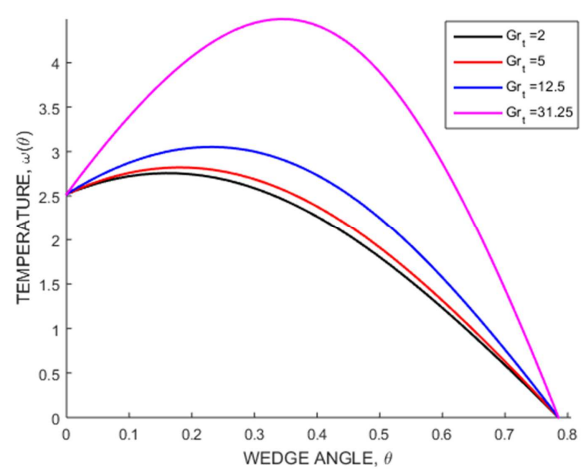

Figure 10. Temperature profiles for different values of the Grashof Temperature number.



Figure 11. Magnetic Induction profiles for different values of the Grashof Temperature number. 


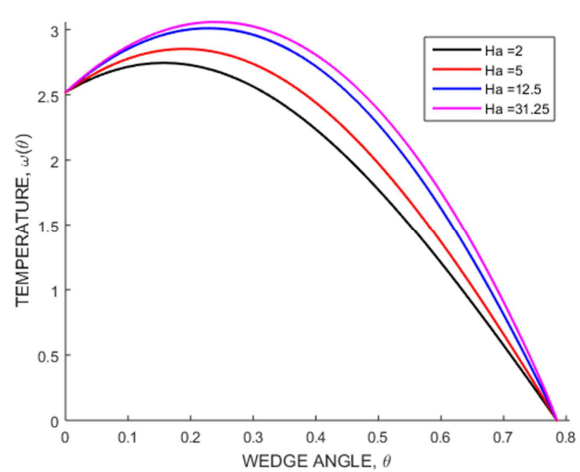

Figure 12. Temperature profiles for different values of the Hartmann number.

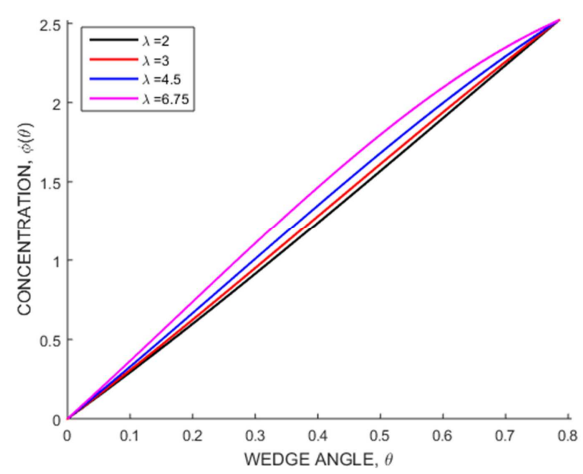

Figure 13. Concentration profiles for different values of the Unsteadiness Parameter.

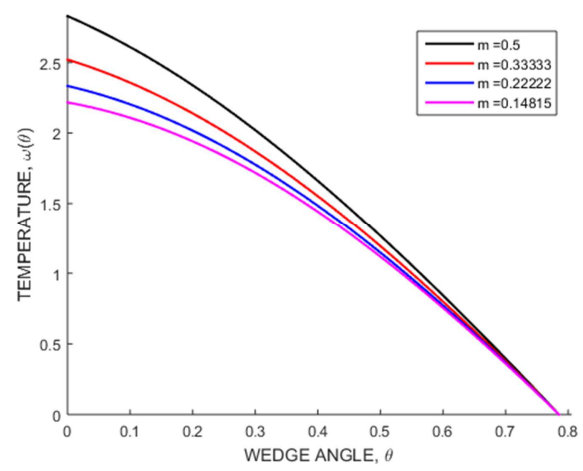

Figure 14. Temperature profiles for different values of the wedge angle Parameter.

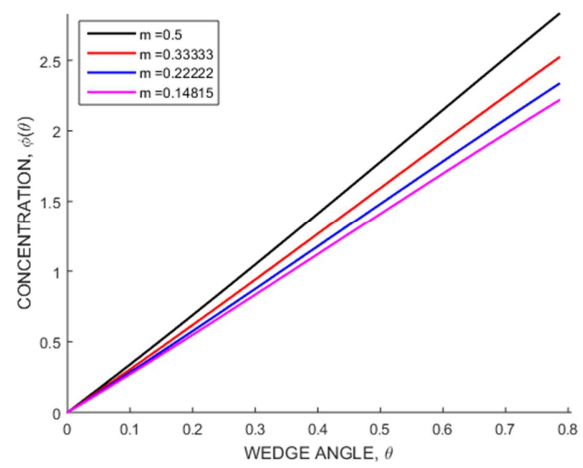

Figure 15. Concentration profiles for different values of the wedge angle
Parameter.

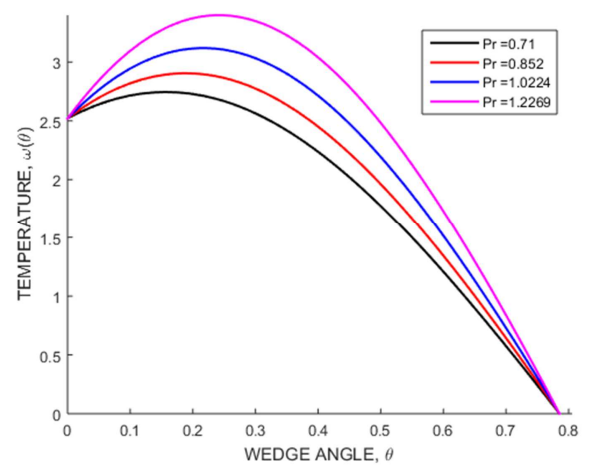

Figure 16. Temperature profiles for different values of the Prandtl.

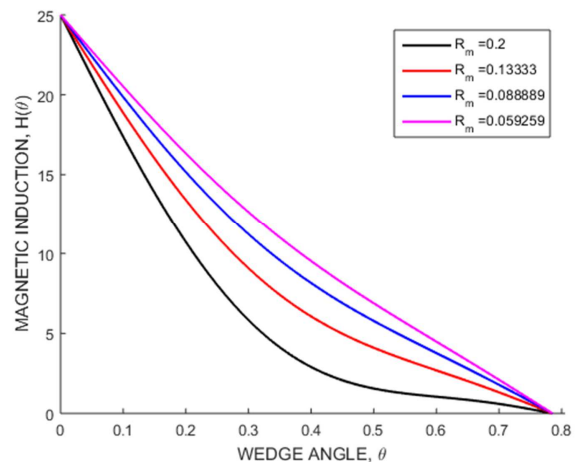

Figure 17. Graph of Magnetic induction profiles while varying Reynold Magnetic number.

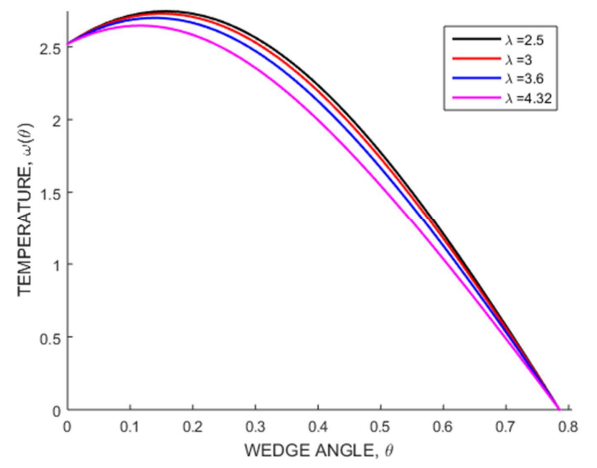

Figure 18. Temperature profiles for different values of Unsteadiness Parameter.

From figure 2, temperature increased with the increasing the suction parameter. Suction increases the velocity of the fluid which in turn leads to increased kinetic energy which leads to increase in temperature due to conversion of kinetic energy to thermal energy. For figure 3, temperature falls as injection parameter increases. Injection results in reduction of the fluid velocity as a result of the boundary layer thickening in turn reducing kinetic energy lowering its conversion to thermal energy thus reducing fluid temperature. Concentration of the fluid decreases with the increase in the Reynolds number as observed from figure 4. This can be alluded to the fact that as the Reynolds number increases, the fluid temperature increases which in turn leads to a decrease 
in the concentration. An increase in temperature leads to increased kinetic energy in the fluid leading to increased vibrations of the molecules of the fluid hence an increase in the distance or spaces between molecules resulting in low concentration. From the graph in figure 5, as the Eckert number is increased, the temperature also increases. Increasing Eckert number leads to a rise in the kinetic energy leading to increased vibration of the molecules which results in conversion of kinetic energy to heat energy hence increase in temperature. From figure 6, increasing Grashof concentration number, magnetic induction increases. This is because increasing Grashof concentration number causes the viscous forces to reduce thus increasing the fluid velocity thus increasing magnetic induction. Temperature increased with Grashof concentration number increased form figure 7. Grashof concentration number increasing, leads to a reduction in the viscous forces and meanwhile with viscosity and temperature inversely associated, a decrease in the viscous forces results in temperature increase. Increasing the Grashof concentration number as in figure 8 , the fluid velocity increased. The viscous forces decreased with the increase in the Grashof concentration number reducing the consequence of the drag force in the fluid hence increased velocities. The velocity increases with the increase in the Grashof Temperature number as shown in figure 9. As the Grashof Temperature number increases, the effect of the viscous drag on the fluid reduces hence the velocity increase. Increasing the Grashof temperature number leads to increasing temperature. As the Grashof Temperature number increases, the viscous drag decreases leading to temperature increase. The magnetic induction increases with the increase in the Grashof Temperature number as shown in figure 11. As Grashof Temperature number increases, the viscous drag decreases which result is increased velocities hence magnetic induction is increased. In figure 12, increasing the Hartman number led to increased temperature. With the Hartmann number increasing, viscous drag decreases and with an inverse relation for temperature and viscosity, there is increase in temperature. From figure 13, the concentration increases with increasing the unsteadiness parameter. As the unsteadiness parameter increases, the boundary wall thickens which results in the increase in concentration since the concentration at the wall is greater than the concentration away from the wall hence an increase in the concentration parameter. From figure 14, the temperature decreases with the decrease in the wedge angle parameter $m$, which decreases the wedge angle $\alpha$. For various $m$ values, i.e.

$$
\begin{gathered}
m=\frac{1}{2}=0.5, \alpha=\frac{\pi}{3}=60^{0}, m=\frac{1}{3}=0.33 \ldots, \alpha=\frac{\pi}{4}=45^{0}, \\
m=\frac{1}{4}=0.25, \alpha=\frac{\pi}{5}=36^{0}, m=\frac{1}{5}=0.2, \alpha=\frac{\pi}{6}=30^{0} . \text { As }
\end{gathered}
$$

the wedge angle decreases, boundary layer effect becomes more pronounced in between the divergent walls and since $T_{w}<T_{\infty}$, the temperature decreases from $T_{\infty}$ to $T_{w}$. The fluid concentration decreases with reduction in wedge angle $\alpha$ as shown in figure 15 . As the wedge angle decreases, boundary layer effect becomes prominent in the region of the flow with the walls moving towards the center line and since $C_{w}>C_{\infty}$ with the concentration of wall greater than the concentration at the centerline. As the wedge angle decreases, the velocity of the fluid increases hence reduced concentration from figure 16, the temperature increased with the Prandtl number increase. With Prandtl number increased, viscous forces effects dominates the fluid flow and since viscosity and temperature are inversely related, an increase in the viscous forces led to decreased temperature.

From the graph on figure 17, the magnetic induction increases with increasing Reynolds magnetic number. There is increase in the velocity of the fluid with increasing Reynolds magnetic number resulting in increased interactions between the fluid and the magnetic field hence increasing magnetic induction.

Temperature decreases with the increasing unsteadiness parameter as observed from the graph on figure 18. As the unsteadiness parameter increases, boundary layer thickens and since the wall temperature is less than the free stream temperature, $T_{w}<T_{\infty}$, the effect of the wall increases in the flow region hence decrease in the temperature.

\section{Conclusion}

The unsteady Jeffrey-Hamel flow in the presence of the inclined magnetic field with suction and injection has been investigated and the effect of various parameters discussed. In conclusion, the temperature increases with the increase in the suction parameter, Eckert number, Grashof Temperature number, Hartmann number, wedge angle, and the Prandtl number but decreases with the increase in the injection parameter. The concentration of the fluid increases with the increase in the unsteadiness parameter and the wedge angle parameter while it decreases with the increase in the Reynolds number. Magnetic induction and velocity increase with the increase in the Grashof temperature and concentration numbers. The temperature decreases with time while the magnetic induction increases with the increase in the magnetic Reynolds number.

\section{Nomenclature}

$\begin{array}{ll}\rho & \text { The density of the fluid } \\ r & \text { Radius of channel } \\ \theta & \text { The angle of the channel } \\ \alpha & \text { Half angle of the channel } \\ H & \text { Inclined Magnetic field } \\ u_{r}, u_{\theta} & \text { Velocity along } r, \theta \text { direction } \\ \beta & \text { Inclination angle of magnetic field } \\ T & \text { Temperature } \\ t & \text { Time } \\ \Phi & \text { Viscous dissipation function } \\ \mathbf{H} & \text { Induced Magnetic field } \\ \mathbf{B} & \text { Total magnetic field } \\ \mu & \text { Dynamic viscosity } \\ K & \text { Thermal diffusivity } \\ \sigma & \text { Electrical conductivity }\end{array}$




$\begin{array}{ll}P r & \text { Prandtl number } \\ \operatorname{Re} & \text { Reynold number } \\ R_{m} & \text { Reynold magnetic number } \\ E c & \text { Eckert number } \\ J & \text { Joule heating parameter } \\ H a & \text { Hartmann number } \\ G_{r(C)} & \text { Grashof number for Concentration } \\ G_{r(T)} & \text { Grashof number for Temperature }\end{array}$

\section{References}

[1] Jeffery, G. B. "L. The two-dimensional steady motion of a viscous fluid." The London, Edinburgh, and Dublin Philosophical Magazine and Journal of Science 29, no. 172 (1915): 455-465.

[2] Hamel, Georg. "Spiralförmige Bewegungen zäher Flüssigkeiten." Jahresbericht der Deutschen mathematikerVereinigung 25 (1917): 34-60.

[3] W. I. Axford, "The magnetohydrodynamic Jeffrey-Hamel problem for a weakly conducting fluid," The Quarterly Journal of Mechanics and Applied Mathematics, vol. 14, pp. 335-351, 1961.

[4] Imani, A. A., Rostamian, Y., Ganji, D. D., \& Rokni, H. B. (2012). Analytical investigation of Jeffery-Hamel flows with high magnetic field and nanoparticle by rvim.

[5] Koeltzsch, K., Dinkelacker, A., \& Grundmann, R. (2002). Flow over convergent and divergent wall riblets. Experiments in fluids, 33(2), 346-350.

[6] Makinde, O. D. and P. Y. Mhone (2006). Hermite- Pade' Approximation approach to Hydromagnetic flows in convergent-divergent channels. Applied Mathematics and Computations 181(2), 966-972.

[7] Esmaili, Q., A. Ramiar, E. Alizadeh and D. D. Ganji (2008). An approximation of the analytical Solution of the JefferyHamel flow by decomposition method. Physics Letters $A$ $372,3434-3439$.

[8] T. Hayat, F. M. Abbasi, M. Al-Yami, S. Monaquel, Slip and Joule heating effects in mixed convection peristaltic transport of nanofluid with Soret and Dufour effects, J. Mol. Liq. 194 (2014) 93-99.

[9] E. M. Abo-Eldahab, M. Abd El-Aziz, Blowing/suction effect on hydromagnetic heat transfer by mixed convection from an inclined continuously stretching surface with internal heat generation/absorption, Int. J. Therm. Sci. 43 (2004) 709-719.

[10] A. J. Chamkha, Double-diffusive convection in a porous enclosure with cooperating temperature and concentration gradients and heat generation or absorption effects, Numer. Heat Transfer, Part A: Appl. 41 (1) (2002) 65-87.

[11] M. Umamaheswar, S. V. K. Varma and M. C. Raju, Unsteady MHD free convective visco-elastic fluid flow bounded by an infinite inclined porous plate in the presence of heat source, viscous dissipation, and Ohmic heating, International journal of advanced science and technology, 61 (2013) 39-52.

[12] Vijayakumar, K., \& Reddy, E. K. (2017). MHD Boundary Layer Flow of a VISCO-Elastic Fluid Past a Porous Plate with Varying Suction and Heat Source/Sink in the Presence of Thermal Radiation and Diffusion. Global Journal of Pure and Applied Mathematics, 13(6), 2717-2733

[13] Zubair Akbar, M., Ashraf, M., Farooq Iqbal, M., \& Ali, K. (2016). Heat and mass transfer analysis of unsteady MHD nanofluid flow through a channel with moving porous walls and medium. AIP Advances, 6(4), 045222.

[14] Pourabdian, M., Qate, M., Morad, M. R., \& Javareshkian, A. (2016). The Jeffery-Hamel flow and heat transfer of nanofluids by homotopy perturbation method and Comparison with Numerical Results. arXiv preprint arXiv:1601.05298.

[15] Meher, R., \& Patel, N. D. (2019). A study on magnetohydrodynamic Jeffery-Hamel flows with heat transfer problem in Eyring-Powell fluid using Differential Transform Method. Journal of Applied Mathematics and Computational Mechanics, 18(3).

[16] Ara, A., Khan, N. A., Naz, F., Raja, M. A. Z., \& Rubbab, Q. (2018). Numerical simulation for Jeffery-Hamel flow and heat transfer of micropolar fluid based on differential evolution algorithm. AIP Advances, 8(1), 015201.

[17] Ochieng, F. O., Kinyanjui, M. N., \& Kimathi, M. E. (2018). Hydromagnetic Jeffery-Hamel Unsteady Flow of a Dissipative Non-Newtonian Fluid with Nonlinear Viscosity and Skin Friction. Global Journal of Pure and Applied Mathematics, 14(8), 1101-1119.

[18] Alam, M. S., Haque, M. M., \& Uddin, M. J. (2016). The convective flow of nanofluid along with a permeable stretching/shrinking wedge with second-order slip using Buongiorno's mathematical model. International Journal of Advanced in Applied Mathematics and Mechanics, 3(3), 7991.

[19] Sattar, M. A. (2013). Derivation of the similarity equation of the 2-D unsteady boundary layer equations and the corresponding similarity conditions. American Journal of Fluid Dynamics, 3(5), 135.

[20] Nagler, J. (2017). Jeffery-Hamel flow of non-Newtonian fluid with nonlinear viscosity and wall friction. Applied Mathematics and Mechanics, 38(6), 815-830.

[21] Alam, M. S., \& Huda, M. N. (2013). A new approach for local similarity solutions of an unsteady hydromagnetic free convective heat transfer flow along a permeable flat surface. International Journal of Advances in Applied Mathematics and Mechanics, 1(2), 39-52.

[22] Alam, M. D. S., Khan, M. A. H., \& Alim, M. A. (2016). Magnetohydrodynamic Stability of Jeffery-Hamel Flow using Different Nanoparticles. Journal of Applied Fluid Mechanics, 9(2).

[23] Ojiambo, V., Kinyanjui, M., \& Kimathi, M. (2018). A study of two-phase Jeffery Hamel flow in a geothermal pipe. 